\title{
Addendum: Improved global fit to non-standard neutrino interactions using COHERENT energy and timing data
}

\author{
Pilar Coloma, ${ }^{a}$ Ivan Esteban, ${ }^{b}$ M.C. Gonzalez-Garcia ${ }^{b, c, d}$ and Michele Maltoni ${ }^{e}$ \\ ${ }^{a}$ Instituto de Física Corpuscular, Universitat de València and CSIC, \\ Edificio Institutos de Investigación, Calle Catedrático José Beltrán 2, E-46980 Valencia, Spain \\ ${ }^{b}$ Departament de Física Quàntica $i$ Astrofísica and Institut de Ciències del Cosmos, \\ Universitat de Barcelona, Diagonal 647, E-08028 Barcelona, Spain \\ ${ }^{c}$ Institució Catalana de Recerca i Estudis Avançats (ICREA), \\ Pg. Lluis Companys 23, E-08010 Barcelona, Spain \\ ${ }^{d}$ C.N. Yang Institute for Theoretical Physics, Stony Brook University, \\ Stony Brook, NY 11794-3840, U.S.A. \\ e Instituto de Física Teórica UAM/CSIC, Universidad Autónoma de Madrid, \\ Calle de Nicolás Cabrera 13-15, Cantoblanco, E-28049 Madrid, Spain \\ E-mail: pcoloma@ific.uv.es, ivan.esteban@fqa.ub.edu, \\ maria.gonzalez-garcia@stonybrook.edu, michele.maltoni@csic.es
}

AdDEndum TO: JHEP02(2020)023

ArXiv EPRINT: 1911.09109 

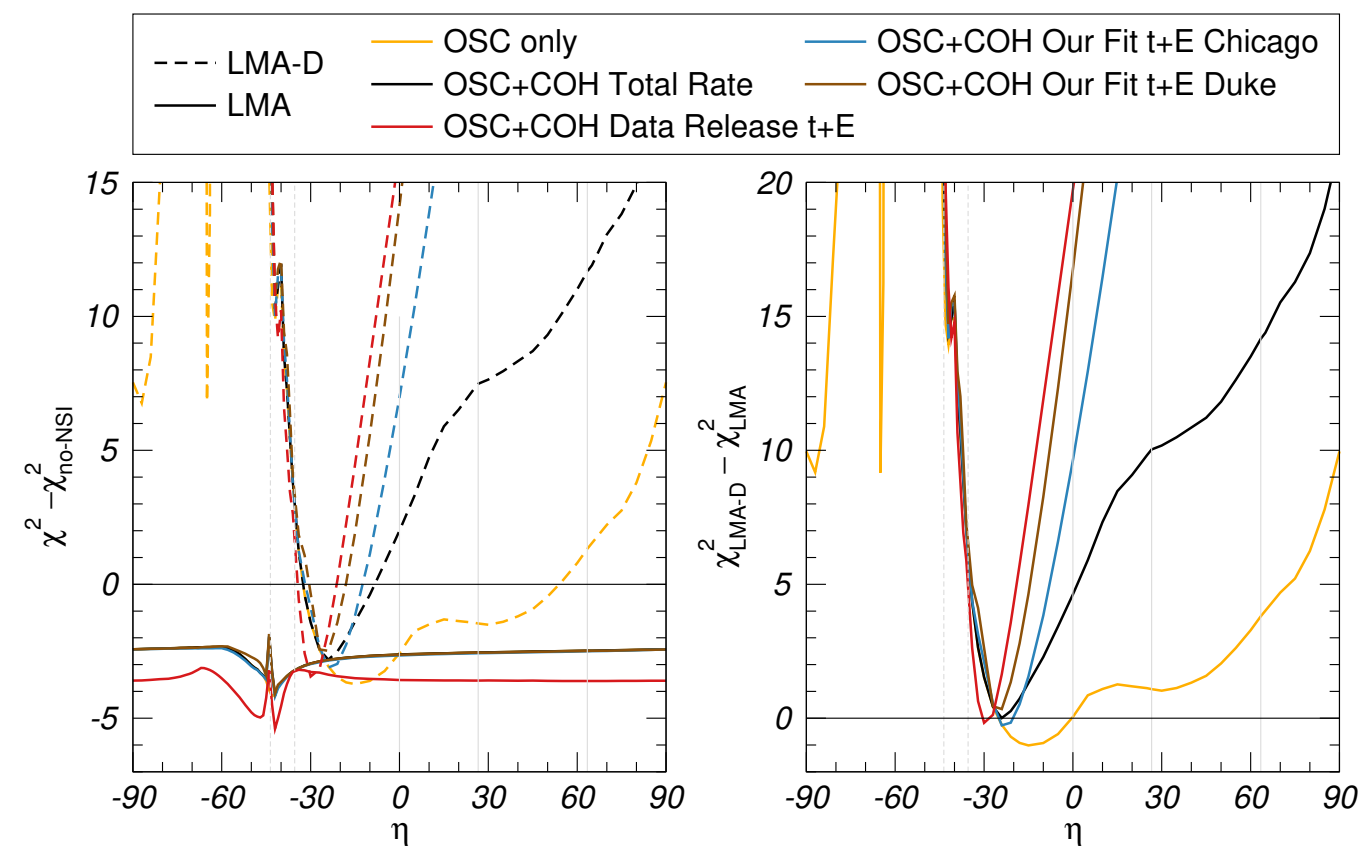

Figure 1. Left: $\chi_{\text {LMA }}^{2}(\eta)-\chi_{\text {no-NSI }}^{2}$ (full lines) and $\chi_{\text {LMA-D }}^{2}(\eta)-\chi_{\text {no-NSI }}^{2}$ (dashed lines) for the analysis of different data combinations (as labeled in the figure) as a function of the NSI quark coupling parameter $\eta$. All solid lines but the red one falls on top of each other. Right: $\chi_{\text {dark }}^{2}-\chi_{\text {light }}^{2} \equiv$ $\chi_{\text {LMA-D }}^{2}(\eta)-\chi_{\text {LMA }}^{2}(\eta)$ as a function of $\eta$. See text for details.

In this addendum we re-assess the constraints on Non-Standard Interactions (NSI) from the combined analysis of data from oscillation experiments and from COHERENT after including the new data released since the publication of ref. [1], in particular the new results presented at the Neutrino2020 conference. New data considered includes the latest total energy spectrum and the day-night asymmetry of the SK4 2970-day sample presented at Neutrino2020 [2], and the latest results from long-baseline (LBL) experiments T2K $[3,4]$ and NOvA $[5,6]$. In addition, we have updated the reactor experiments DoubleChooz $[7,8]$ to $1276 / 587$ days of far/near detector data and RENO $[9,10]$ to 2908 days of exposure.

The main effect driven by the new results concerns the analysis of solar oscillation data. The quantification of the effects in the oscillation analysis has been presented in a separate Addendum to ref. [11]. Here we quantify the induced changes in the results of the combined analysis of oscillation data with COHERENT results, which were contained in section 4.2 of ref. [1]. In particular we present in figures 1 and 2 the new version of figures 8 and 9 , and in table 1 the new version of table 2 .

In brief, in the left panel in figure 8 of ref. [1] we found that the introduction of NSI lead to a substantial improvement of the fit already for the LMA solution (solid lines) with respect to the oscillation data analysis, resulting in a sizable decrease of the minimum $\chi_{\text {LMA }}^{2}$ with respect to the standard oscillation scenario. This was driven by a well-known tension at the level of $\Delta \chi^{2} \sim 7.4$ between solar and KamLAND data in the determination of $\Delta m_{21}^{2}$. Correspondingly the inclusion of NSI improved the combined fit by about $2.2 \sigma$ 
over a broad range of values of $\eta$. As discussed in ref. [12], with the updated SK4 solar data the tension between the best fit $\Delta m_{21}^{2}$ of KamLAND and that of the solar results has decreased to $\Delta \chi_{\text {solar }}^{2}=1.3$. So now in the left panel in figure 1 we see that for the LMA solution the combined global fit with NSI leads to a decrease of about 2 units in $\chi^{2}$ for most values of $\eta$ and for most variants of the $\mathrm{COH}$ analysis. The only exception is the analysis of the combination with COHERENT t+E data using the data release assumptions, for which including NSI can improve the fit in LMA by $\Delta \chi^{2} \sim 4$ for most values of $\eta$.

Concerning the status of the LMA-D degeneracy, we find that when COHERENT total rate results are taken into account and we include the new oscillation data, LMA-D is allowed below $3 \sigma$ with respect to LMA for a slightly wider range of values of $\eta$. This is a consequence of the increase of $\chi_{\text {LMA }}^{2}$. Quantitatively, LMA-D is now allowed at $3 \sigma$ for values of $\eta$ in the following ranges:

$$
\begin{array}{rlrl}
-37^{\circ} \lesssim \eta \lesssim 20^{\circ} & & \text { COHERENT Total Rate, } \\
-37^{\circ} \lesssim \eta \lesssim-14^{\circ} & \text { COHERENT t+E Data Release, } \\
-37^{\circ} \lesssim \eta \lesssim 0^{\circ} & & \text { COHERENT t }+ \text { E Our Fit Chicago }, \\
-37^{\circ} \lesssim \eta \lesssim-9^{\circ} & & \text { COHERENT t }+ \text { E Our Fit Duke }
\end{array}
$$

Figure 2 contains the updated $\Delta \chi^{2}$ profiles for each of the six NSI coefficients after marginalization over the undisplayed oscillation parameters and the other five NSI coefficients not shown in a given panel, for three representative cases of NSI models including couplings to up quarks only, down quarks only and to protons. The corresponding $2 \sigma$ ranges are also provided in table 1 for convenience. The main difference introduced by the new oscillation data is that now the two minima corresponding to the degeneracy on $\varepsilon_{\mu \mu}^{\mathrm{coh}}$ obtained after the inclusion of timing information for COHERENT, is no longer broken after combination with the updated oscillation data. This leads to the appearance of disconnected allowed ranges at $2 \sigma$ when comparing table 1 with table 2 in ref. [1]. We notice also that at the confidence level shown no LMA-D solution for these specific cases is allowed.

From figure 1 we see that best fit within LMA-D solution is achieved for $\eta \sim-30^{\circ}$, which corresponds to a model with couplings to up and down quarks in a ratio $\sim-1.2$. Assuming the same coupling normalization employed for the cases of $\varepsilon_{\alpha \beta}^{u}$ and $\varepsilon_{\alpha \beta}^{d}$ shown in table 1 , the $2 \sigma$ allowed ranges for $\varepsilon_{\alpha \beta}^{\eta}$ (with $\eta=-30^{\circ}$ ) from our global fit including the time and energy information of COHERENT with the Duke quenching factor read

\begin{tabular}{|l|c|c|}
\hline & \multicolumn{1}{|c|}{ wihtin LMA } & within LMA-D \\
\hline$\varepsilon_{e e}^{\eta}$ & {$[-0.132,+0.949] \oplus[+3.169,+4.134]$} & {$[+0.752,+1.661]$} \\
$\varepsilon_{\mu \mu}^{\eta}$ & {$[-0.178,+0.576] \oplus[+3.108,+3.873]$} & {$[+3.368,+3.994]$} \\
$\varepsilon_{\tau \tau}^{\eta}$ & {$[-0.180,+0.570] \oplus[+3.110,+3.869]$} & {$[+3.373,+3.999]$} \\
$\varepsilon_{e \mu}^{\eta}$ & {$[-0.158,+0.075]$} & {$[+0.016,+0.132]$} \\
$\varepsilon_{e \tau}^{\eta}$ & {$[-0.304,+0.316]$} & {$[-0.105,+0.127]$} \\
$\varepsilon_{\mu \tau}^{\eta}$ & {$[-0.015,+0.027]$} & {$[-0.010,+0.008]$} \\
\hline
\end{tabular}



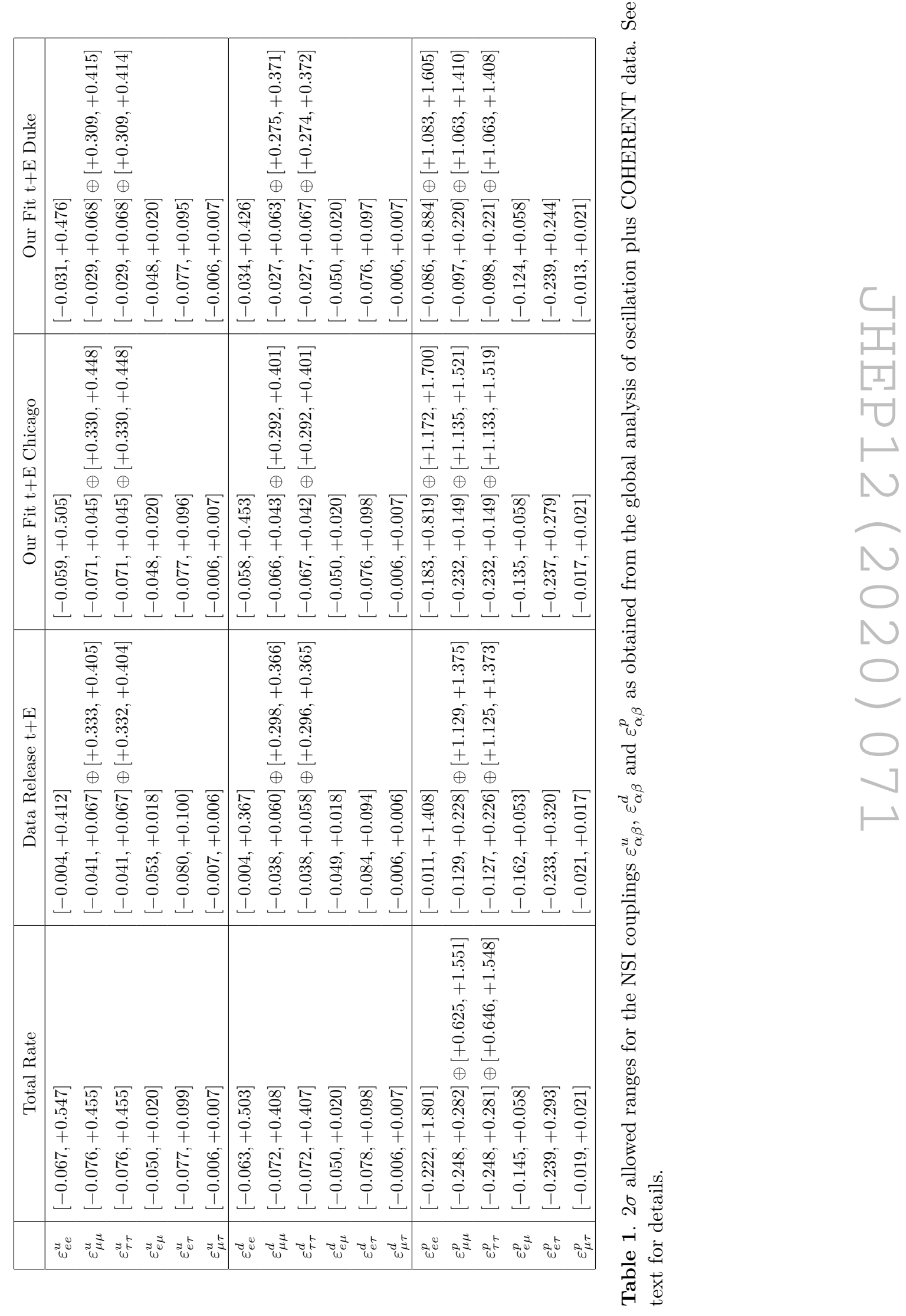

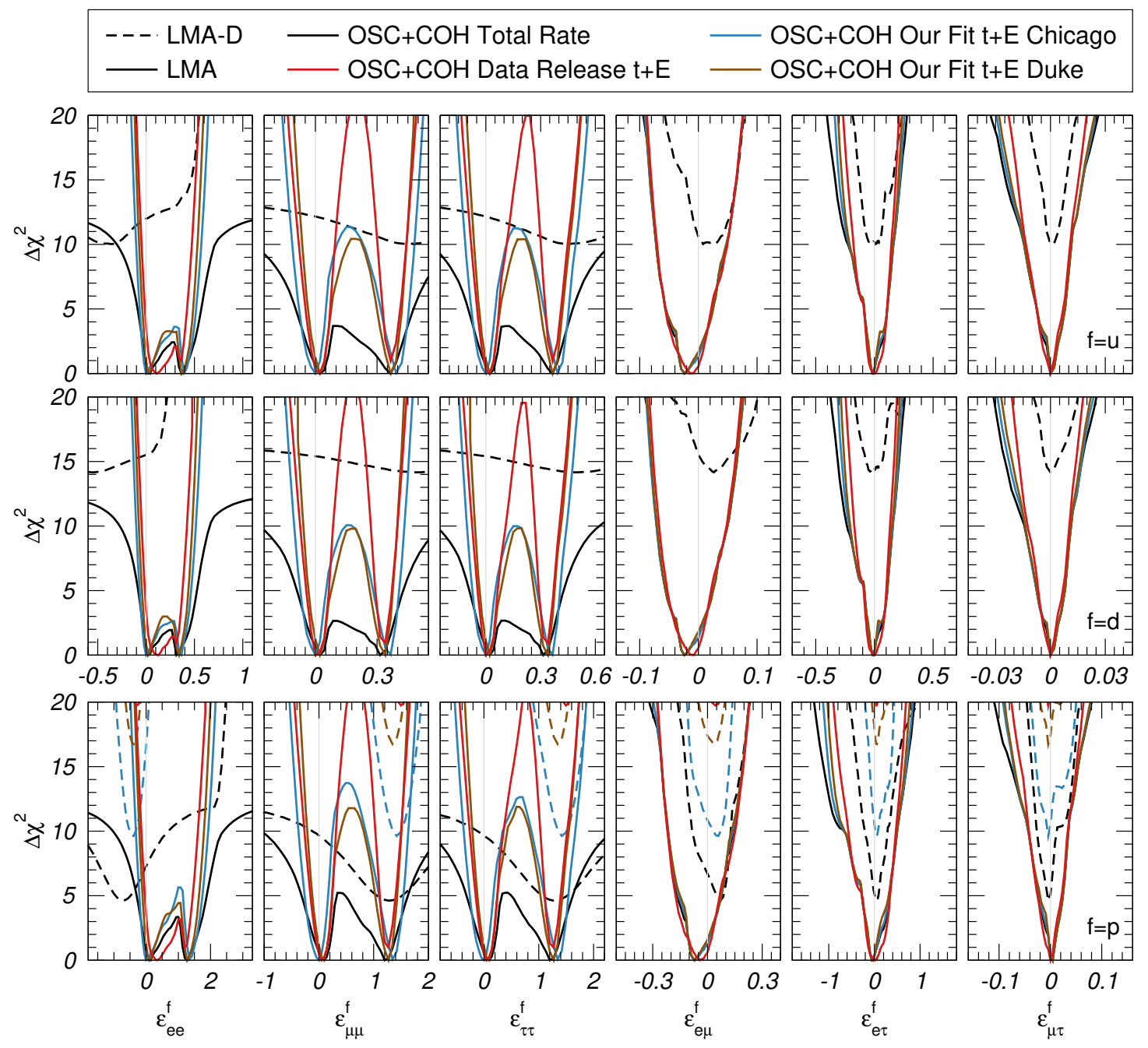

Figure 2. Dependence of the $\Delta \chi_{\text {global }}^{2}$ function on the NSI couplings with up quarks (upper row), down quark (central row) and, protons (lower row) for the global analysis of oscillation and COHERENT data. In each panel $\chi_{\text {global }}^{2}$ is marginalized with respect to the other five NSI couplings not shown and with respect to the oscillation parameters for the LMA (solid) and LMA-D (dashed) solutions. The different curves correspond to the different variants of the COHERENT analysis implemented in this work: total rate (black), $\mathrm{t}+\mathrm{E}$ Data Release (red), $\mathrm{t}+\mathrm{E}$ with $\mathrm{QF}-\mathrm{C}$ (blue), and $\mathrm{t}+\mathrm{E}$ with QF-D (brown); see text for details.

In summary, we find that the main effect of the inclusion of the new data - characterized by a smaller tension between the solar and KamLAND results - is the reduction of the improvement of the quality of the fit with NSI with respect to that of pure oscillations within the LMA solution. Conversely LMA-D becomes allowed for a slightly wider range of the $\eta$ parameter, i.e., for a slightly broader variety of models with NSI couplings to combinations of up and down quarks.

\section{Acknowledgments}

This work was supported by the spanish grants FPA2016-76005-C2-1-P, FPA2016-78645-P, PID2019-105614GB-C21 and PID2019-110058GB-C21, by USA-NSF grant PHY-1915093, 
by AGAUR (Generalitat de Catalunya) grant 2017-SGR-929, by EU ITN project H2020MSCA-ITN-2019/870881-HIDDeN, and by the Spanish Agencia Estatal de Investigación through the grant "IFT Centro de Excelencia Severo Ochoa SEV-2016-0597". IE acknowledges support from the FPU program fellowship FPU15/0369. The authors acknowledge use of the HPC facilities at the IFT (Hydra cluster).

Open Access. This article is distributed under the terms of the Creative Commons Attribution License (CC-BY 4.0), which permits any use, distribution and reproduction in any medium, provided the original author(s) and source are credited.

\section{References}

[1] P. Coloma, I. Esteban, M.C. Gonzalez-Garcia and M. Maltoni, Improved global fit to non-standard neutrino interactions using COHERENT energy and timing data, JHEP 02 (2020) 023 [arXiv: 1911.09109] [INSPIRE].

[2] Y. Nakajima, SuperKamiokande, talk given at the XXIX International Conference on Neutrino Physics and Astrophysics, June 22-July 2, Chicago, U.S.A. (2020), available on on Zenodo.

[3] T2K collaboration, Constraint on the matter-antimatter symmetry-violating phase in neutrino oscillations, Nature 580 (2020) 339 [Erratum ibid. 583 (2020) E16] [arXiv: 1910.03887] [INSPIRE].

[4] P. Dunne, Latest Neutrino Oscillation Results from T2K, talk given at the XXIX International Conference on Neutrino Physics and Astrophysics, June 22-July 2, Chicago, U.S.A. (2020), available on on Zenodo.

[5] NOvA collaboration, First measurement of neutrino oscillation parameters using neutrinos and antineutrinos by NOvA, Phys. Rev. Lett. 123 (2019) 151803 [arXiv:1906.04907] [INSPIRE].

[6] A. Himmel, New oscillation results from the NOvA experiment, talk given at the XXIX International Conference on Neutrino Physics and Astrophysics, June 22-July 2, Chicago, U.S.A. (2020), available on on Zenodo.

[7] Double CHOOZ collaboration, Double CHOOZ $\theta_{13}$ measurement via total neutron capture detection, Nature Phys. 16 (2020) 558 [arXiv:1901.09445] [INSPIRE].

[8] T. Bezerra, New results from the Double Chooz experiment, talk given at the XXIX International Conference on Neutrino Physics and Astrophysics, June 22-July 2, Chicago, U.S.A. (2020), available on on Zenodo.

[9] RENO collaboration, Measurement of reactor antineutrino oscillation amplitude and frequency at RENO, Phys. Rev. Lett. 121 (2018) 201801 [arXiv:1806.00248] [INSPIRE].

[10] J. Yoo, RENO, talk given at the XXIX International Conference on Neutrino Physics and Astrophysics, June 22-July 2, Chicago, U.S.A. (2020), available on on Zenodo.

[11] I. Esteban, M.C. Gonzalez-Garcia, M. Maltoni, I. Martinez-Soler and J. Salvado, Updated Constraints on Non-Standard Interactions from Global Analysis of Oscillation Data, JHEP 08 (2018) 180 [arXiv: 1805.04530] [INSPIRE].

[12] I. Esteban, M.C. Gonzalez-Garcia, M. Maltoni, T. Schwetz and A. Zhou, The fate of hints: updated global analysis of three-flavor neutrino oscillations, JHEP 09 (2020) 178 [arXiv: 2007.14792] [INSPIRE]. 